\title{
Fibonacci Backoff Algorithm for IEEE 802.15.4/ZigBee
}

\author{
Muneer Bani Yassien, Marwa Salayma, Wail Mardini, Yaser Khamayseh \\ Department of Computer Science, \\ Jordan University of Science and Technology, Irbid (Jordan) \\ Tel: +962-7201000Ｅ-mail: masadeh@just.edu.jo
}

Received: July 12, 2012 Accepted: August 29, 2012 Published: September 29, 2012

DOI: 10.5296/npa.v4i3.2094

URL: http://dx.doi.org/10.5296/ npa.v4i3.2094

\begin{abstract}
The IEEE 802.15.4 is a standard for Wireless Personal Area Network (PAN) that supports low data rate, low cost, low complexity and low power consumption applications. The CSMA/CA algorithm of the IEEE 802.15.4 MAC layer employs the Binary Exponential Backoff (BEB) function to compute the backoff delay for each node. Using BEB function, it is possible that two or more nodes may collide if they choose the same backoff exponent value. Consequently this will increase collision and network contention level which will degrade the network overall performance. To overcome this problem, this paper proposes a Fibonacci Backoff (FIB) function to compute the backoff interval. In FIB, each node shall wait for an incremental backoff periods as they need to access the channel. The performance of FIB algorithm is compared against the BEB function.
\end{abstract}

Keywords: Backoff algorithm, Fibonacci, IEEE 802.15.4, Medium access control, Performance analysis.

\section{Introduction}

The IEEE 802.15.4 standard was proposed and has been commercially adopted to specify protocols for the physical and data link layers. The standard is basically proposed for two devices communication and is considered as promising for low-cost, low-power and low data rate networks like Wireless Sensor Networks (WSNs) [1].

The MAC layer of IEEE 802.15.4 standard works in two possible modes; the beacon and 
beaconless modes and supports both star and peer to peer network topologies [2]. In beacon enabled mode the central coordinator is responsible for sending beacons in order to synchronize nodes [2]. Two successive beacons contain between them what is called a superframe [2]. This superframe is divided into 16 active period time slots along with an optional inactive period in which all nodes can sleep together in the case that sleep mode is enabled. The active period in turn consists of a Contention Access Period (CAP) and an optional Contention Free Period (CFP) [2].

In order to avoid all nodes transmit at the same time, a commonly followed approach in CAP is the slotted Carrier Sense Multiple Access/ Collision Avoidance (CSMA/CA) mechanism through which nodes can compete with each other's when accessing a channel, whereas in beaconless enabled mode it follows the un-slotted CSMA/CA [2]. In either case, the IEEE 802.15.4 based devices shall wait for a randomly chosen backoff period before they start emitting data [2]. This period is chosen randomly from rang of $\left[0-2^{\mathrm{BE}}-1\right]$ where $\mathrm{BE}$ represents the backoff exponent which is needed to find the period through which a node should stay silent aside before trying to assess the medium. The value of BE can be initialized to $0,1,2$ or 3 but the default is set to 3 and in case the channel sensed busy this value shall increase to the maximum value of 5 [3]. This backoff delay time along with channel sensing and finally packet transmission are all happen in the backoff period which is bounded by the boundaries of each slot contained in the 16 superframe slots [3].

However, following CSMA/CA backoff strategy cannot totally avoid collisions. This is due to the fact that the backoff period is randomly chosen from the small range of $\left[0-2^{\mathrm{BE}}-1\right]$ and hence, there is a great possibility that more than two nodes pick up or reach the same backoff periods and as the network becomes larger in scale or nodes emit intensive and frequent traffic loads, this case is more likely to happen [3]. Obviously, this situation makes those nodes which become silent for the same backoff periods detect that the availability of free channel simultaneously. As a result, they start emitting data at the same time causing unavoidable collision. This situation leads to retransmission and consequently will degrade power consumption that is a critical constraint in WSN. This will also affect network throughput which is a result of packet losses, and hence affect network performance [3].

If we analyze the backoff mechanism carefully, we can conclude that the random nature of the binary exponential backoff (BEB) scheme is the main cause of such a problem. Hence, unless a variation to such mechanism is done, the problem will continue to happen. However, many backoff mechanism modifications have been proposed in literature to accommodate and solve such situation, but they all still follow the BEB strategy. Previous works methods vary between changing the value of $\mathrm{BEB}$ parameters according to some conditions and improving the channel crier sensing (CCA) schemes [3]. Obviously, the root responsible cause of the problem which is the randomness chosen period from the insufficient distributed numbers in the small range still exists.

Hence, a new backoff mechanism is needed that does not depend on random chose of backoff periods. One suggested way to do so is to follow the Fibonacci Increment, a well known mathematical series that is formulated incrementally as each new subsequent number is formulated by adding the direct previous two numbers [4].

The incremental behavior of Fibonacci mechanism is inspired from the Fibonacci backoff 
algorithm proposed by Manaseer [4] for IEEE 802.11 standard based networks and avoids two or more nodes to choose the same backoff period and hence avoid collision caused by BEB mechanism [4].

The following section gives an overview of some backoff schemes proposed in literature. The third section clarifies the new FIB algorithm scheme. The fourth section illustrates some basic calculations and theoretical scenarios. Simulation set up along with results achieved are illustrated in section 5 after which the paper work is concluded in section 6 .

\section{Related Work}

This section lists some backoff schemes proposed in literature which all make variations and improvements on the current IEEE 802.15.4 binary exponential backoff parameters in a way or another. However, the last three algorithms concern the IEEE 802.11 backoff standard followed by MANET from which the new Fibonacci algorithm proposed in this work is inspired.

Rao and Marandin (2006) [3], proposed an Adaptive Backoff Exponent (ABE) algorithm through which Backoff exponents (BE) values can be managed based on a node's contribution to the network traffic. The algorithm implements a variable macMinBE and adopted only by nodes involved in the transmission activity. ABE algorithm aimed at solving the possibility of nodes choosing the same BE values caused basically by the few number of possible Bes. Consequently, nodes will backoff identical time periods causing collisions. ABE cannot support large scale PAN's.

Lee and Ryu (2011) [5], set the value of BE in the slotted CSMA/CA to an efficient Backoff Exponent (EBE) variable. EBE value is initialized according to the number of nodes in the PAN. As the number of nodes joining the network decreasing, it is rational to lessen the nodes backoff delay periods. This can be accomplished by initializing EBE to the minimum value between macMinBE variable and 2 while the battery life extension (BLE) in the beacon frame is set to 1 . On the contrary, collisions and channel contention shall increase as the number of nodes joining the network increases and the need for longer backoff periods arises. This can be accomplished by setting EBE to the average of the number of backoff delay periods by the number of nodes which are joining the network. As the value of EBE is set, it is given to BE and nodes backoff according to this value as usual. Simulation results reveal that adopting EBE increases throughput while decreases energy consumption and network load.

Khan et al (2010) [6], proposed the Improved BEB (IBEB) algorithm. IBEB minimizes the possibility that nodes may pick the same BE values and hence waiting for the same backoff time periods. In [6] nodes uses another value to calculate the backoff periods rather than choosing only BE randomly. They chose Interim Backoff (IB) value between $10 \%$ and $40 \%$ of the calculated backoff time and they also used the unit Interim Period (IP) to decrease the probability of choosing both BE and IB. Simulation results revealed that IBEB outperforms the BEB scheme when tested on different network load and scale.

Lee et al. (2009) [7], proposed a backoff technique named Non- Overlapping Binary Exponential Backoff (NO-BEB) that aimed at decreasing collision level in large scale PANs. 
NO-BEB calculates data delivery failure in order to distinguish the range of the current backoff periods from passed ones and uses this information to fairly distribute the random backoff delay and hence, decreasing the possibility of nodes choosing the same backoff periods according to failure counts.

Khanafer et al (2012) [8], proposed the Priority-Based BEB (PB-BEB) that takes nodes channel access priority under consideration. PB-BEB follows a probabilistic estimation that exploits the information of channel collision level in order to manage the number of $C C A \mathrm{~s}$. Higher priorities are given to those nodes that obliged to backoff multiple time periods as they are trying to access the channel while nodes that just begin competing on the channel are given less priorities. PB-BEB utilizes channel at the cost of increasing delay.

Khanafer et al (2011) [9], proposed an Adaptive Backoff Algorithm (ABA) that aims at maximizing channel utilization through exploiting nodes experienced collisions information in order to probabilistically estimate the appropriate size of the contention window $(\mathrm{CW})$. In case of high level collisions, nodes should backoff from sending packets. Whereas if collisions level decrease, large backoff periods should be avoided in order to exploit idle channel and consequently improving channel utilization.

Lee and Wu (2009) [10], proposed the Delayed Backoff Algorithm (DBA). [10] Suggests coordinator to assign backoff time periods for nodes that need to access channel frequently, hence, those nodes need not choose random backoff time value. The importance of such enhancement arises as the network traffic load increases. In such a case, DBA proved to maximize delivery ratio, throughput and energy efficiency. DBA can be implemented for the mesh and large scale multi-hop networks.

Wong et al (2010) [11], proposed the Additional Carrier Sensing (ACS) algorithm aims at maximizing channel utilization. A new clear channel assessment (CCA) referred to as CCA3 is proposed to check if it is possible to access the channel after receiving the acknowledgment for previously transmitted packets and if so, then, this will avoid the need of sending further future CCAs and further backoff delays. Through this technique, the channel is utilized as it exploits the implicit information concluded as the channel detected busy when sending the previous two CCAs.

Woo et al. (2008) [12], proposed the Knowledge-based Exponential Backoff (KEB) algorithm. KEB exploits nodes local information about channel collisions level in order to improve channel utilization. To estimate collision level, KEB follows the principle of Exponential Weighted Moving Average (EWMA). BE will increase or decrease according to the collision level which by its turn will go above or beyond a previously specified threshold. Markov Chain model was adopted to analyze KEB and simulation results indicate that it outperforms BEB in terms of throughput.

Ko. et al (2006) [13], suggested an enhancement of the IEEE 802.15.4 MAC through proposing a new state transition scheme for the aim of minimizing the backoff periods for nodes that access the channel frequently by dynamically adjusting the minBE value to be minimum than that of the default which is originally specified to 3 . Simulation revealed that nodes with low minBE value have much better throughput than those with static minBE value of 3 .

Haas et al. (2003) [14], proposed the Sensing Backoff Algorithm (SBA) that aims at 
improving medium throughput and achieving fairness when accessing the channel randomly. By adopting the SBA, each node chooses its backoff delay time dynamically according to the feedback concerning the channel activities. SBA calculates backoff periods as a function of the node transmission time when the active node senses the channel idle. SBA was compared to both BEB and MILD backoff algorithms and simulation run on a fully connected network and results revealed that SBA outperformed both of them.

Ha et al. (2007) [15], proposed two mechanisms that enhance both the BE values and backoff periods. An Enhanced Collision Resolution (ECR) mechanism have been proposed to exploit channel contention information sensed through the two CCA in order to specify the BE. The Enhanced Backoff (EB) mechanism was proposed to exploit CCA results in order to avoid unnecessary backoffs delays by shifting the range of backoff periods.

Jin et al. (2010) [16], enhances the IEEE 802.15.4 backoff algorithm through following the concept of game theory. The basic idea is that if a node is frequently access the channel and successfully transmits packets, then its backoff delay decreases slowly instead of performing CCR immediately. Simulation results show that the proposed algorithm decreases collision level and increase throughput in wireless sensor networks.

Song et al. (2007) [17], propose the New Adaptive CSMA/CA Algorithm (NACA). NACA aims at decreasing energy consumption in WSN through reducing redundant transmission. The proposed algorithm monitors the channel traffic and dynamically derives the backoff parameters in such a way that reduces collision and redundant retransmission and thus preserving power which is a critical issue in WSN.

Manaseer et al (2006) [4], proposed the Fibonacci Increment Backoff (FIB) scheme for mobile ad hoc networks that follow IEEE 802.11 standard. Incremental behaviour of FIB minimizes the large range between consecutive contention window sizes. Simulation results revealed that FIB performance outperforms the traditional exponential backoff performance as it increases throughput.

Bani Yassein et al (2009) [18], proposed a Pessimistic Linear-Exponential Backoff (PLEB) algorithm for mobile ad hoc networks (MANET) which follow the IEEE 802.11 standard. PLEB merges Exponential backoff scheme with linear backoff scheme and exploits the advantages of both algorithms. Exponential increments reduce redundant transmissions and so has the advantage if increasing network throughput, whereas linear increment decreases the transmission delay.

Manaseer et al (2011) [19], worked on improving the medium access control of IEEE 802.11 standard. They studied the effect of adopting different backoff schemes which are the linear, Pessimistic Linear Exponential and logarithmic increments to contention windows as opposed to the affect of using BEB scheme which increases contention window sizes exponentially. Their investigation and simulation results revealed that choosing the suitable backoff increments depends on network status and choosing the right algorithm increases network throughput.

\section{The Proposed Fibonacci Algorithm (FIB)}

This work proposes a new IEEE 802.15.4/ZigBee backoff strategy that allows nodes to wait for an incremental period formulated from the mathematical Fibonacci series when 
applying CSMA/CA algorithm as they need to access the channel. The Fibonacci Increment is a well known mathematical series that is formulated incrementally as each new subsequent number is formulated by adding the direct previous two numbers according to (1): [4]

$$
F(x)=F(x-2)+F(x-1), \text { where } \mathrm{x}>=0, \quad F(0)=0 \text { and } F(1)=1
$$

Figure 1 shows a pseudo code for the proposed FIB algorithm where $\mathrm{n}$ is the maximum Fibonacci sequence number to be chosen, in other words, it is the range size FIB algorithm will go through. BP refers to the backoff period which typically equals to $0.00032 \mathrm{~s}$.

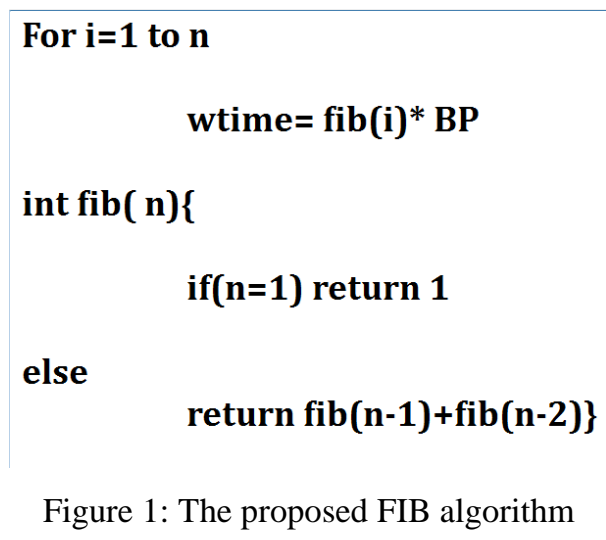

The incremental behavior of Fibonacci mechanism is expected to minimize the possibility that two or more nodes choose the same backoff period in dense networks or networks with intensive traffic loads and hence avoid collision caused by BEB mechanism; this will be clarified through an example in the next section.

\section{Theoretical Analysis}

Before going through our experiments; some calculations have to be investigated and that is what this section shows.

As it stated previously; in order to save power which is the primary constrain for which the IEEE 802.15.4 is proposed, nodes are allowed to choose a random backoff period from a small range of $\left[0-2^{\mathrm{BE}}-1\right]$. For this reason, the $\mathrm{BE}$ value of $\mathrm{BEB}$ algorithm is allowed to reach 5 and not more, otherwise, longer values will lead to longer waiting time and longer delay which will directly drain the power causing dissipation which contradicts the IEEE 802.15.4 design goals. So, the BEB time each node shall wait before starting transmission can be calculated according to (2).

$$
\text { wtime }=\text { rand }^{*} B P
$$

Where wtime is the waiting time each node shall wait, rand is the random value chosen from the range of $\left[0-2^{\mathrm{BE}}-1\right]$ and $\mathrm{BP}$ refers to the backoff period which equals to $0.32 \mathrm{~ms}$. According to the previous formula, the $\mathrm{BEB}$ minimum wtime $=0$, while the maximum $=31$ * $0.32=9.92 \mathrm{~ms}$.

Regarding to FIB algorithm, the maximum backoff period nodes may reach depends on the range of Fibonacci series chosen for FIB algorithm. Table 1 shows the maximum backoff 
periods in ms for 10 different Fibonacci series numbers (range of values).

Table 1: Maximum backoff time for different FIB ranges

\begin{tabular}{|l|l|l|l|l|l|l|l|l|l|l|}
\hline FIB Range & 1 & 2 & 3 & 4 & 5 & 6 & 7 & 8 & 9 & 10 \\
\hline Fibonacci Value & 1 & 1 & 2 & 3 & 5 & 8 & 13 & 21 & 34 & 55 \\
\hline Maximum BP(ms) & 0.32 & 0.32 & 0.64 & 0.96 & 1.6 & 2.56 & 4.16 & 6.72 & 10.88 & 17.6 \\
\hline
\end{tabular}

Now, let us assume a network of 6 nodes and at the first time unit (t1) all the 6 nodes try to transmit packets simultaneously, then the FIB backoff periods will be distributed amongst the 6 nodes as Table 2 depicts where each column concerns a different FIB maximum sequence value the network shall reach. However, In order to eliminate nodes start with identical backoff periods; FIB algorithm is allowed to start working through a Fibonacci series with a range of size $2(\mathrm{fib}=2)$.

Table 2: Backoff period's distribution for six nodes under 10 FIB ranges at the first time unit

\begin{tabular}{|l|l|l|l|l|l|l|l|l|l|}
\hline Node/BP & fib=2 & fib=3 & fib=4 & fib=5 & fib=6 & fib=7 & fib=8 & fib=9 & fib=10 \\
\hline node1 & 1 & 1 & 1 & 1 & 1 & 1 & 1 & 1 & 1 \\
\hline node2 & 1 & 2 & 2 & 2 & 2 & 2 & 2 & 2 & 2 \\
\hline node3 & 1 & 1 & 3 & 3 & 3 & 3 & 3 & 3 & 3 \\
\hline node4 & 1 & 2 & 1 & 5 & 5 & 5 & 5 & 5 & 5 \\
\hline node5 & 1 & 1 & 2 & 1 & 8 & 8 & 8 & 8 & 8 \\
\hline node6 & 1 & 2 & 3 & 2 & 1 & 13 & 13 & 13 & 13 \\
\hline
\end{tabular}

Table 2 tells us that at t1, if the network is allowed to work through FIB sequence number with a maximum value of 2 (fib=2), then all the 6 nodes would have the same backoff periods whereas for fib $=7,8,9$ and fib $=10$, no repeated value would occur.

Table 3: A scenario for FIB behaviour followed by 6 nodes

\begin{tabular}{|c|c|c|c|c|c|c|c|}
\hline fib=7 & node1 & node2 & node3 & node4 & node5 & node6 & Network status \\
\hline $\mathrm{t} 1$ & 1 & 2 & 3 & 5 & 8 & 13 & \\
\hline $\mathrm{t} 2$ & transmit & 1 & 2 & 4 & 7 & 12 & $\begin{array}{c}\text { successful } \\
\text { transmission }\end{array}$ \\
\hline $\mathrm{t} 3$ & 1 & transmit & 1 & 3 & 6 & 11 & $\begin{array}{c}\text { successful } \\
\text { transmission }\end{array}$ \\
\hline $\mathrm{t} 4$ & transmit & 2 & transmit & 2 & 5 & 10 & collision \\
\hline $\mathrm{t} 5$ & 3 & 1 & 5 & 1 & 4 & 9 & \\
\hline $\mathrm{t} 6$ & 2 & transmit & 4 & transmit & 3 & 8 & collision \\
\hline $\mathrm{t} 7$ & 1 & 8 & 3 & 13 & 2 & 7 & \\
\hline $\mathrm{t} 8$ & retransmit & 7 & 2 & 12 & 1 & 6 & $\begin{array}{c}\text { successful } \\
\text { retransmission }\end{array}$ \\
\hline $\mathrm{t} 9$ & 1 & 6 & 1 & 11 & transmit & 5 & $\begin{array}{c}\text { successful } \\
\text { transmission }\end{array}$ \\
\hline $\mathrm{t} 10$ & transmit & 5 & retransmit & 10 & 2 & 4 & collision \\
\hline
\end{tabular}


Now, let us see how this scenario works as the time unit grows from t1 to t10 for fib $=7$. We assume a worst case for 6 nodes getting their backoff periods as they all have data ready for transmission. For discussion simplicity, we assume that each time unit equals one bakoff period and at any new time unit, nodes have data ready for transmission and it takes a one time unit for any node to transmit its packet.

It can be noticed from Table 3 that at the first time unit (t1), all the 6 nodes have data ready for transmission and the backoff periods are chosen gradually with no two nodes chose the same waiting time. At the second time unit (t2), node1 has finished its backoff waiting period and finds the channel idle so transmits its packet while other nodes still passing their backoff periods. The same scenario occurs at $\mathrm{t} 3$ at which node 2 has finished its waiting time and finds the channel idle so transmits its packets. Meanwhile, node1 finds the channel busy, so it needs to reinitiate the FIB algorithm and wait for one backoff period again.

The problem starts to arise at the fourth time unit (t4). Both node1 and node 3 have finished their waiting time simultaneously and find the channel idle, so try to transmit together which will cause collision. At the same time, node 2 finished sending its packets and tries to access the channel again but finds it busy so it is obliged to wait for 2 backoff periods (FIB already initiated by node 1 at $\mathrm{t} 3$ ). At $\mathrm{t} 5$, both node1 and node 3 need to retransmit their data again but before doing so both have to backoff for some time again which took its incremental value from a previously initiated FIB sequence assuming that this scenario works in a round robin fashion. The same scenario occurs at $t 6$ as both node 2 and node 4 find the channel idle so try to transmit together causing collision. FIB algorithm continues to behave the same for the next 4 time units with successful transmissions happen at $t 8$ and $t 9$ and a collision occurs at t10. Obviously, retransmission will degrade network performance especially for small FIB ranges.

Let's compare the previous analysis with what would happen as a worst case as applying BEB algorithm by the 6 nodes under the same assumptions as it shown in the debajo de:

Table 4: A scenario for BEB behavior followed by six nodes

\begin{tabular}{|c|c|c|c|c|c|c|c|}
\hline BEB & node1 & node2 & node3 & node4 & node5 & node6 & $\begin{array}{c}\text { Network } \\
\text { status }\end{array}$ \\
\hline $\mathrm{t} 1$ & $2^{3}$ & $2^{3}$ & $2^{3}$ & $2^{3}$ & $2^{3}$ & $2^{3}$ & \\
\hline $\mathrm{t} 7$ & 1 & 1 & 1 & 1 & 1 & 1 & \\
\hline $\mathrm{t} 8$ & transmit & transmit & transmit & transmit & transmit & transmit & collision \\
\hline $\mathrm{t} 9$ & $2^{4}$ & $2^{4}$ & $2^{4}$ & $2^{4}$ & $2^{4}$ & $2^{4}$ & \\
\hline $\mathrm{t} 24$ & 1 & 1 & 1 & 1 & 1 & 1 & \\
\hline $\mathrm{t} 25$ & retransmit & retransmit & retransmit & retransmit & retransmit & retransmit & collision \\
\hline $\mathrm{t} 26$ & $2^{5}$ & $2^{5}$ & $2^{5}$ & $2^{5}$ & $2^{5}$ & $2^{5}$ & \\
\hline $\mathrm{t} 59$ & 1 & 1 & 1 & 1 & 1 & 1 & \\
\hline $\mathrm{t} 60$ & retransmit & retransmit & retransmit & retransmit & retransmit & retransmit & collision \\
\hline
\end{tabular}

Table 4 indicates that as the BEB executes, the BE of all nodes were initialized to the minimum value of 3 , so nodes are to choose a backoff period from the range of $\left[0-2^{3}-1\right]$. The 
worst case of the longest backoff period chosen would be in case that all nodes backoff for 7 time periods. It took them 7 time units to finally finish the boundary of their backoff periods and find the channel idle altogether so try to transmit all at once which will cause a severe collision (we eliminate time delay counting down behaviour for the space consideration).

All the 6 nodes will try to retransmit so would again increment their BE and choose a value from the range of $\left[0-2^{4}-1\right]$. Again, all the 6 nodes would choose the maximum backoff period from that range and hence will backoff for another 15 backoff periods. At t24, all nodes will finish their backoff periods altogether and sense the channel idle so try to transmit simultaneously causing another severe collision at $\mathrm{t} 25$. The same scenario would happen as all nodes try to retransmit and choose the maximum value from the range of [0-2 -1$]$. It took them a 31 backoff periods and eventually will sense the channel idle and hence try to transmit together causing another third severe collision.

Obviously, the previous scenario makes nodes wait for a high fraction of time while all the transmission trails fail. However, the case that all nodes keep choosing the same backoff periods is somehow an excessive imagination as it is not very likely to happen due to the random nature of BEB algorithm. However, it is always the case that some nodes reach up the same backoff period while passing their waiting time. Also it is very likely to happen that nodes reach up the maximum $\mathrm{BEB}$ value early causing them to delay for high fraction of time especially in densely or high traffic load networks.

\section{Simulation Set Up and Results Discussion}

The performance of the original BEB algorithm of the slotted MCSA/CA algorithm in beacon enabled mode of IEEE 802.15.4 was analyzed along with the performance of the new proposed FIB algorithm. The performance comparison is achieved by testing each algorithm on an IEEE 802.15.4 based star topology consists of 8 nodes one hop away from a PANc. Two scenarios were applied in order to test the effect of emitting different traffic load on the both backoff algorithms. In the first scenario, 6 nodes amongst 9 were chosen to send variable bit rate (VBR) traffic to the PANc whereas in the second one, only 3 VBR traffic applications were applied. In both cases, 50 byte traffic is sent with an exponentially distributed inter-arrival time around the mean of 1 second. PANc is allowed to work through a $50 \%$ duty cycle with a beacon order $(\mathrm{BO})=4$ and a superframe order $(\mathrm{SO})=2$. All the simulation parameters are summarized in Table 5. Performance evaluation was achieved using the QualNet simulator which is network evaluation environment software that supports IEEE 802.15.4 standard along with many other different networks standards. The Performance metrics investigated are:

- Energy Consumption: The total sum of transmitting, receiving, idle and sleeping energy in mWh consumed by PAN nodes.

- Throughput: Number of bits passed across the PAN in a second.

- Average End-to-End delay: The average time spent by a packet to travel from the node to the destination PANc. 
Table 5: QualNet simulation parameters for star topology scenarios

\begin{tabular}{|l|l|}
\hline Parameter & Value \\
\hline Simulator & QualNet 5.2 \\
\hline Physical and MAC model & IEEE 802.15 .4 \\
\hline Area & $80 \mathrm{~m} * 80 \mathrm{~m}$ \\
\hline Number of nodes & 9 \\
\hline Transmission range & $10 \mathrm{~m}$ \\
\hline Simulation time & $1000 \mathrm{~s}$ \\
\hline Channel Frequency & $2.4 \mathrm{GHZ}$ \\
\hline Data rate & $250 \mathrm{kbps}$ \\
\hline TX-Power & $0 \mathrm{dBm}$ \\
\hline Energy model & MICAZ \\
\hline Antenna Height & 0.08 \\
\hline Traffic & VBR \\
\hline Payload size & 50 byte \\
\hline Channel Access Mechanism & Slotted MCSA/CA \\
\hline BO and SO & 4,2 respectively \\
\hline
\end{tabular}

\subsection{Evaluating BEB on a Star Topology}

This simulation part aims at evaluating the performance of BEB scheme of MAC IEEE 8021.5.4 on star topology as applying the two scenarios. In the first scenario, a star topology composed of one PANc with 8 reduced functional devices (RFDs) uniformly deployed in an area of $80 \mathrm{~m} \times 80 \mathrm{~m}$ as shown in Figure 2. The centred node is the PANc and a 3 RFD nodes where chosen to send a VBR traffic of a 50 byte payload size all over the simulation period. While in the second one which is depicted in Figure 3, a 6 RFD nodes where chosen to send a VBR traffic of a 50 byte payload size for the same simulation period.

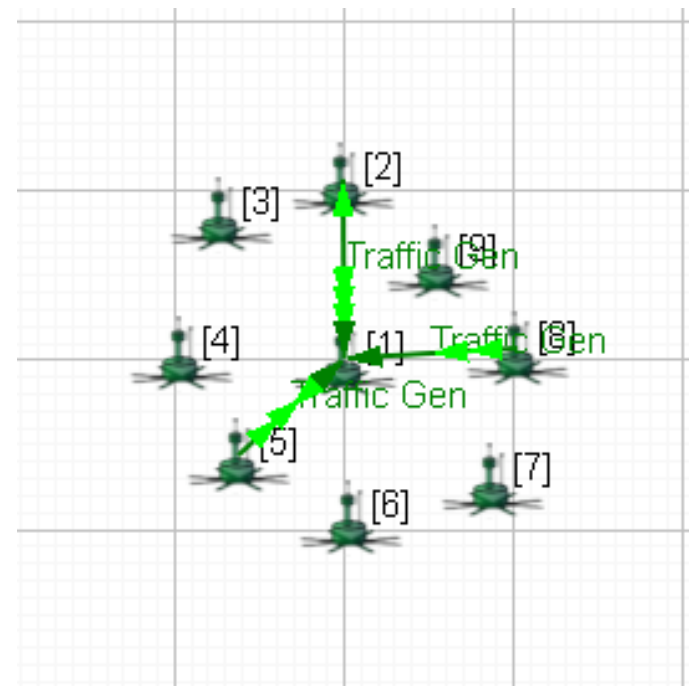

Figure 2: 3 VBR applications execute on a star topology run under QualNet 5.2 


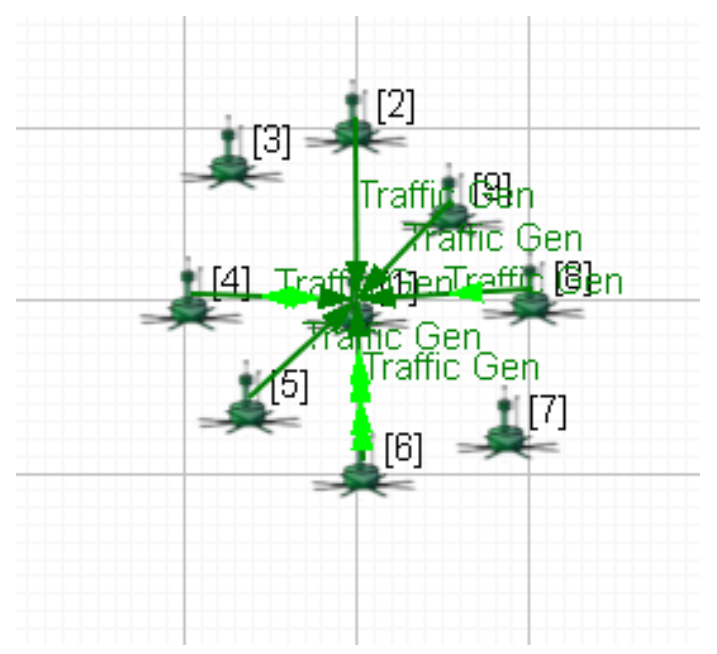

Figure 3: 6 VBR applications execute on a star topology run under QualNet 5.2

\subsection{Evaluating FIB Algorithm on a star topology with Different Fibonacci Sequence Ranges}

The new Fibonacci algorithm was tested on the same topology, the same network scenarios and the same parameters in order to analyze the same metrics. The performance of FIB algorithm is evaluated with different FIB size ranges implemented by variable nodes traffic load in order to locate the best of maximum sequence value. For each range, FIB performance was compared side by side with that of BEB. However, due to the power constraints which is the first design issue IEEE 802.15.4 takes care of and to preserve the same possible range for BEB wtime values; 9 cases for FIB were tested differs in the Fibonacci maximum series value. In other words, series was tested with a minimum backoff period of 2 and allowed to reach up to a maximum of 10 backoff periods.

\subsection{Simulation Results and Discussion}

This subsection depict a comparison between both BEB and FIB algorithms performance according to metrics stated above as running both on a star topology for both scenarios.

a) Average total energy consumption: 


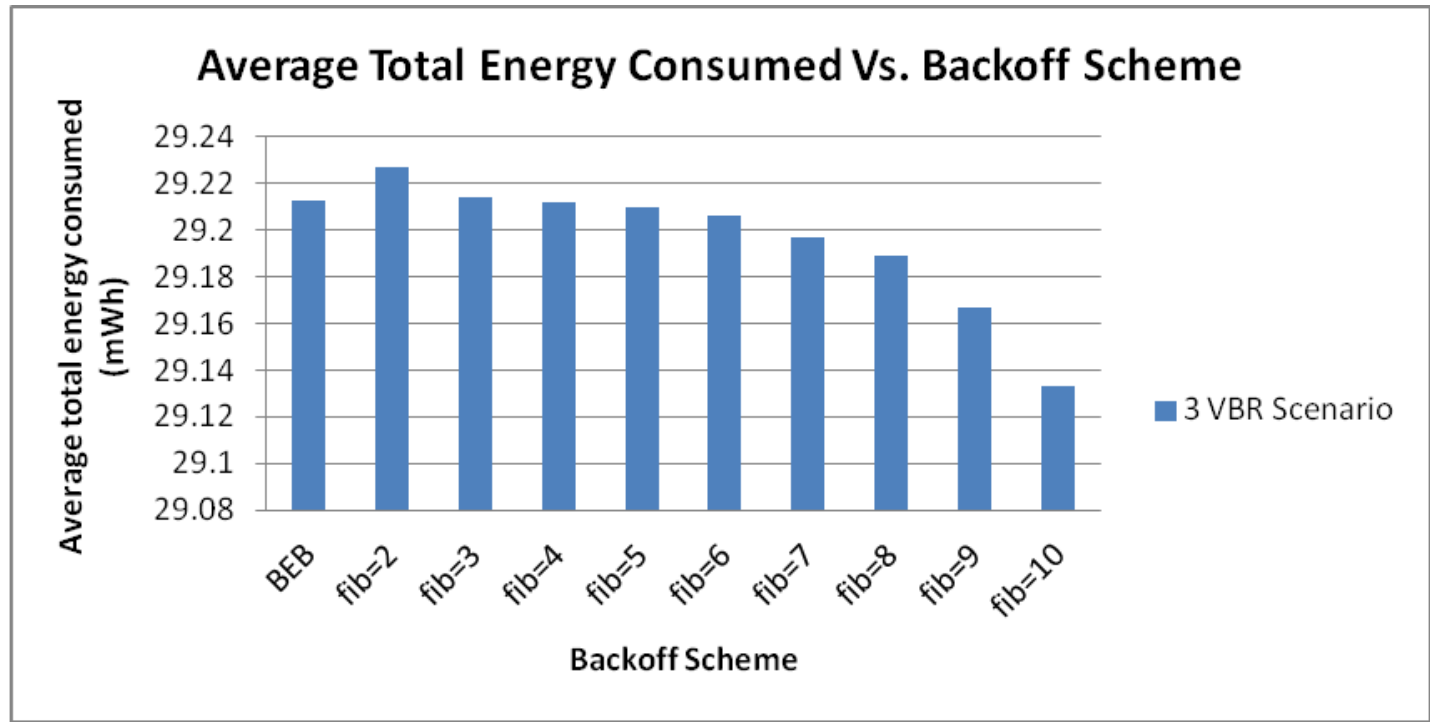

Figure 4: Average total energy consumption vs. backoff algorithm scheme followed by 3 VBR applications

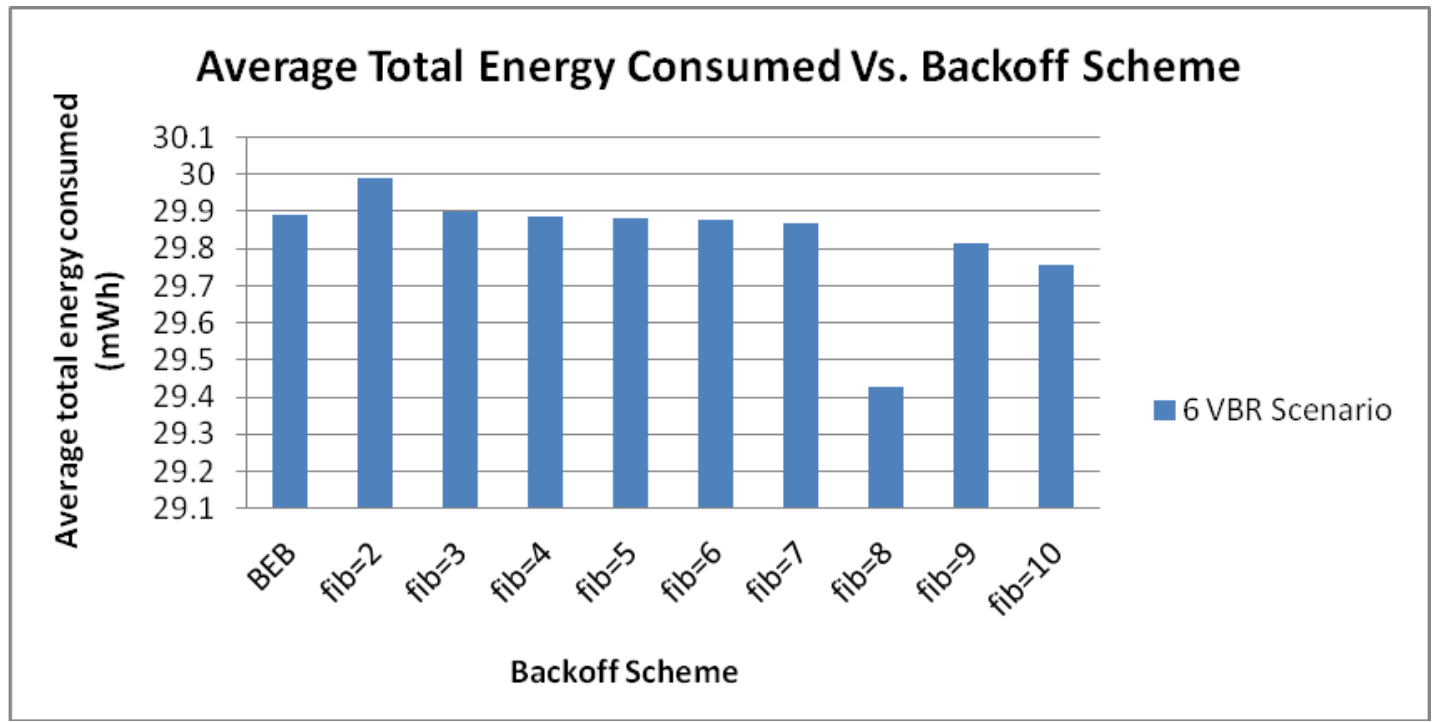

Figure 5: Average total energy consumption vs. backoff algorithm scheme followed by 6 VBR applications

It can be noticed from (Figure 4 and 5) that FIB algorithm outperforms BEB starting from fib $=4$. This note is two folded, less energy is related to less unreasonable waiting time, less collision and good rhythm and synchronization in sending data between nodes which is very likely to happen at fib $=4$ through fib $=8$. The best (short) backoff periods distribution achieved the least energy is actually happen when FIB works through a range of 8 numbers. But unfortunately the happiness never completes, actually less energy at fib $=9$ and 10 is related to a high fraction of delay some nodes would suffer as those nodes are punished with high values of FIB backoff periods causing them to stay idle for long time periods and as we know, idle listening consumes much less energy than both transmission and reception. On the other hand, high energy values at fib $=2$ and fib $=3$ is due to the identical backoff periods nodes shall wait because of the restricted FIB values. In case of fib $=2$ for example, all nodes 


\section{Macrothink}

Network Protocols and Algorithms

ISSN 1943-3581

2012, Vol. 4, No. 3

shall backoff for 1 backoff period which obviously leads to a severe fraction of collisions causing nodes to try retransmit over and over again and hence consuming much power and finally degrading the overall network performance.

Comparing the results with $\mathrm{BEB}$, there is a great possibility that nodes wait unreasonable long bakeoff periods due to high BE values. This case often happens if nodes send packets frequently or have a high traffic load which may cause them reach the maximum BE value rapidly.

b) Average throughput:

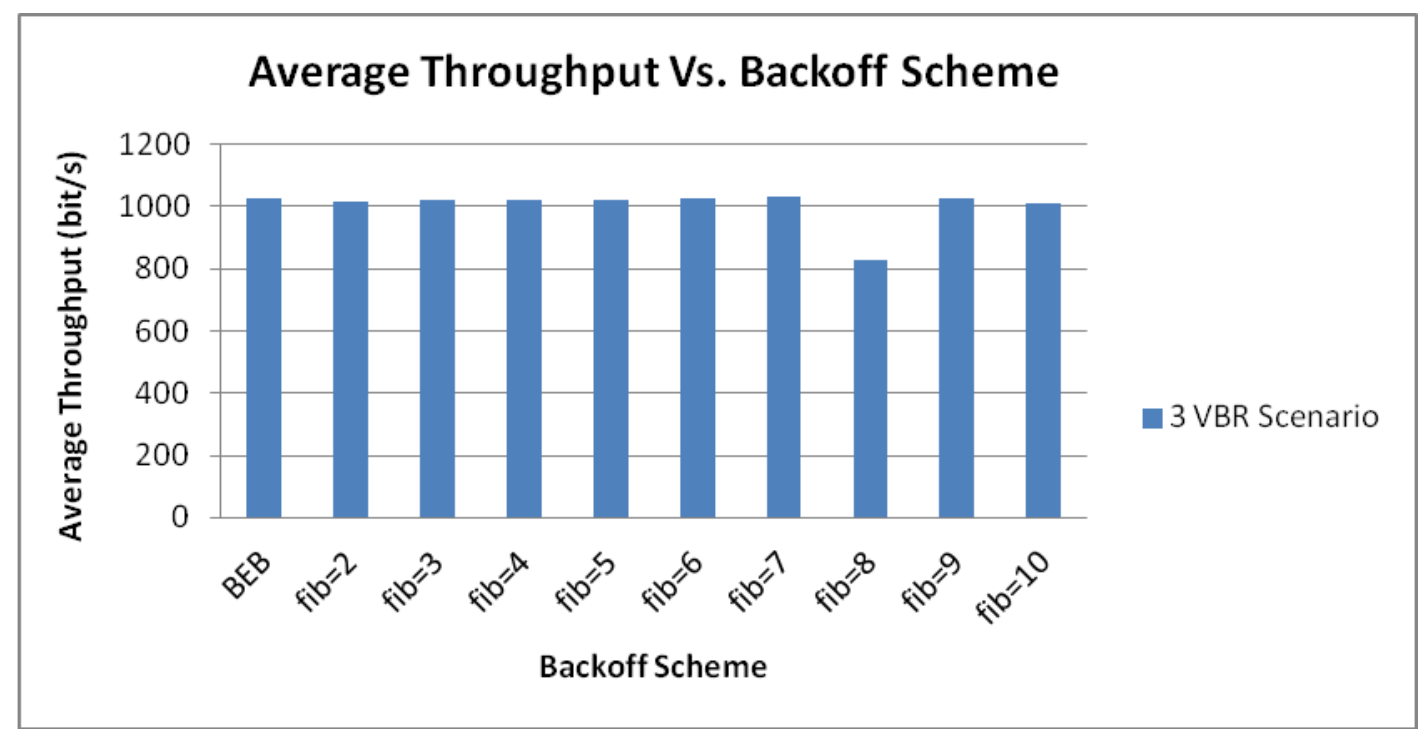

Figure 6: Average throughput vs. backoff scheme followed by 3 VBR applications

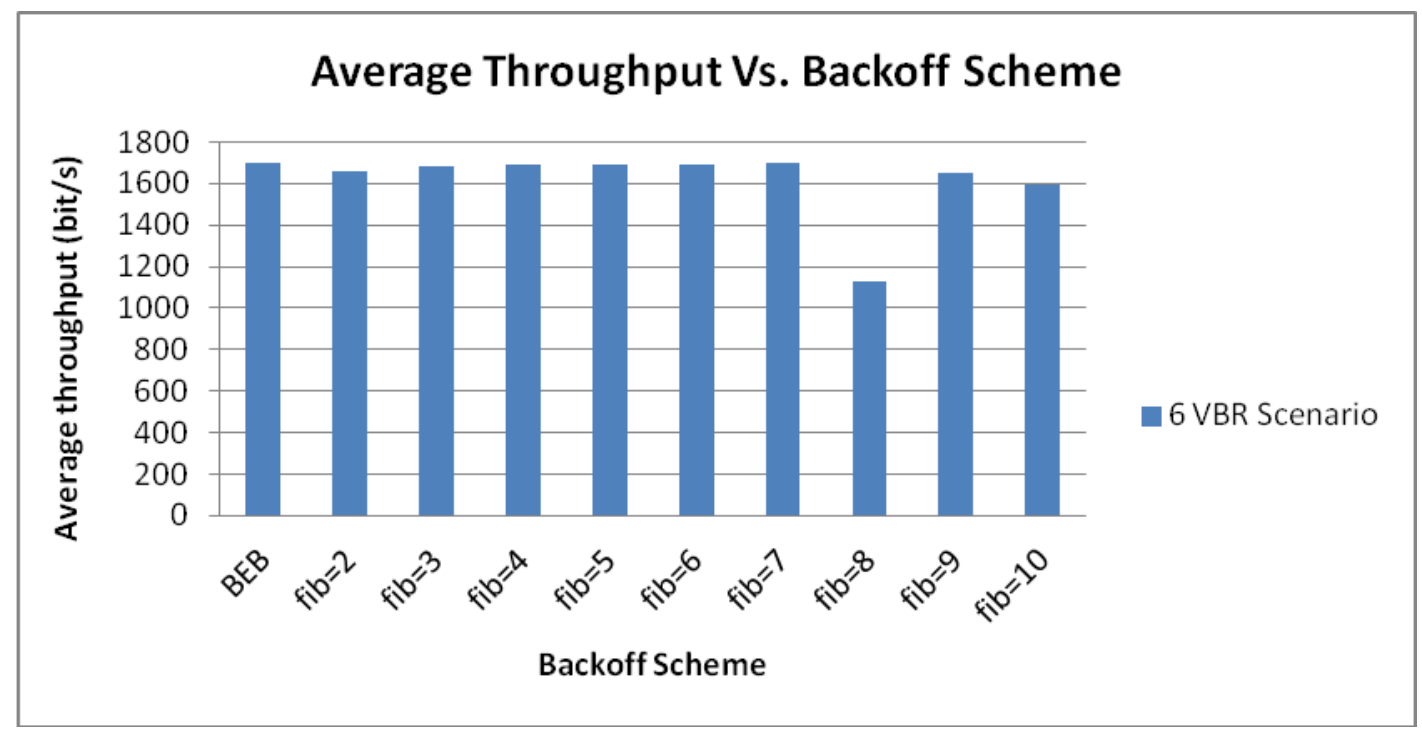

Figure 7: Average throughput vs. backoff scheme followed by 6 VBR applications

In case of 3 VBR applications sent, Figure 6 depicts that FIB algorithm outperforms BEB 


\section{Macrothink}

Network Protocols and Algorithms

ISSN $1943-3581$

2012, Vol. 4, No. 3

at fib $=7$ and acts almost the same as $B E B$ for fib $=4,5,6$ and fib=9. Figure 7 shows that the same pattern happens if $6 \mathrm{VBR}$ applications sent. That's because of the good backoff periods distribution as FIB works through 7 numbers because identical waiting periods are not very likely to happen which is what Table 2 indicates. Obviously, different short backoff periods decrease collisions probability and hence achieve higher throughput. Again, bad results for fib $=2$ and 3 is related to a very high possibility of collisions that may happen as nodes are obliged to wait for the same backoff periods which will forbid them from sending data over and over again till the stage of failure.

c) Average end to end delay:

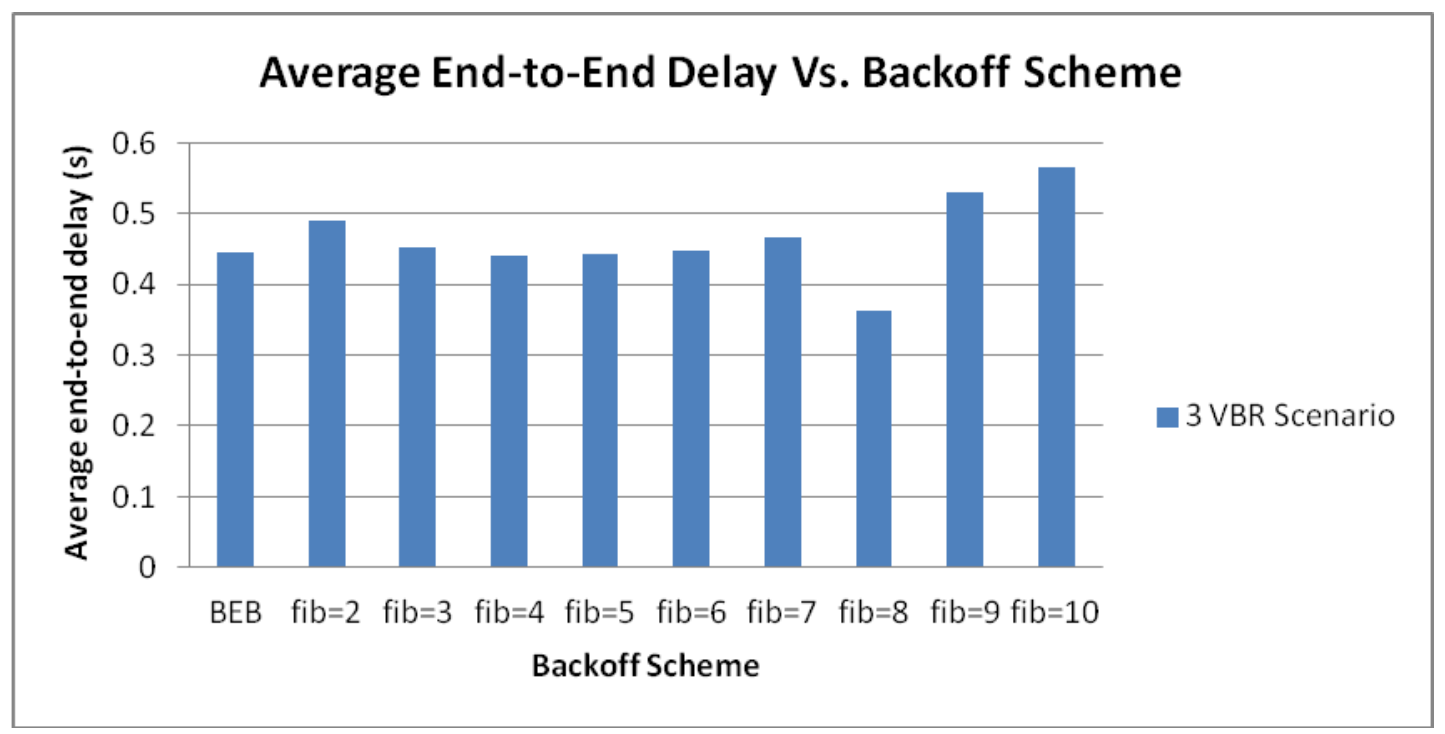

Figure 8: Average end to end delay vs. backoff scheme followed by 3 VBR applications

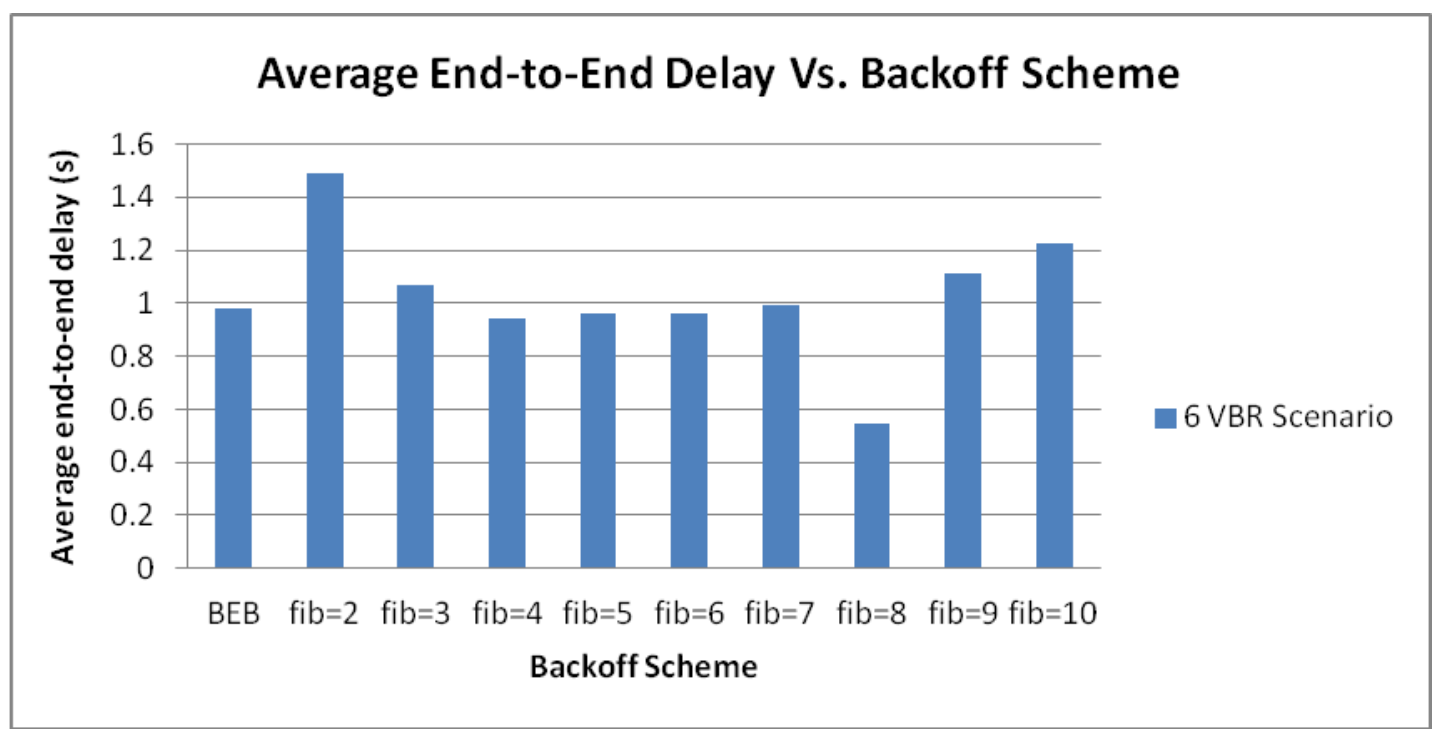

Figure 9: Average end to end delay vs. backoff scheme followed by 3 VBR applications 
(Figure 8, Figure 9) show that FIB scheme outperforms BEB at fib $=4,5$ and $f i b=6$ while acts almost the same at fib=7. This is because of the small wait time values each node is obliged to wait at those FIB values. At fib $=6$ for example, the maximum wait time any node can reach is 8 backoff periods and even if collision happen, nodes re-initiate backoff scheme with small backoff periods all the time, whereas for BEB, it is likely that nodes wait for high backoff periods (31 for example) especially those nodes emit high and frequent traffic rates as they reach their maximum $B E$ value rapidly. However, the high delay values at fib $=9$ and 10 are due to the long backoff periods for high FIB series values. For instance, at fib $=9$, three nodes are likely to be punished and wait for very long backoff periods whereas for fib $=10$, four nodes will do so. This will increase the overall network delay which is directly related to the bad throughput and will decrease power consumption as illustrated previously. On the other hand, for variable bit rate applications, following BEB sounds to be unfair because nodes emitting light traffic load will need to wait unreasonable long time for those nodes which have high traffic load and already reached high BE values to finish transmitting their data. The problem becomes worse as network density increases which degrade the overall performance.

To better summarize results obtained, it is noticed that either for 6 or 3 VBR applications, FIB outperforms BEB at fib=4, 5, 6 and fib $=7$. In our experiments, fib $=7$ gives the higher throughput, less energy and gave almost the same delay. By this, we can conclude that FIB outperforms BEB, but we have to be carful about the size of FIB range we choose at which FIB outperforms BEB. Certainly, this depends on the topology density and traffic rate.

\section{Conclusions and Future Work}

Despite its low complexity and power consumption for which IEEE 802.15.4/ZigBee standard is designed; the BEB scheme followed by CSMA/CA algorithm has the problem of the possibility that nodes pick up or go through the same backoff periods due to the limited $\mathrm{BE}$ values adopted by the standard. This will increase collisions level and consequently degrading the PAN performance. This work proposes and evaluates FIB algorithm to check if it could replace BEB for variable and high traffic load networks. Results show that the proposed FIB outperforms BEB in some cases which depend on the range of the Fibonacci series the algorithm shall follow keeping in mind that FIB range must not exceed 10 numbers in order to conserve energy. Intensive simulation was done on a star topology with 3 and 6 VBR applications in order to choose the best FIB range size after which the overall FIB performance may degrade. It is found that for both the 3 and $6 \mathrm{VBR}$ applications, the maximum Fibonacci series value which achieved the best results was 7. However, results are directly related to the network size and traffic load and it can be said that for large network sizes where no frequent packets transmission, choosing FIB to work through 10 numbers range size seems sufficient as most nodes will backoff for a long time. But for small network sizes where most nodes send packets frequently, it is more proper to let FIB work through a small range ( 4 series numbers for example), this is because active nodes need not wait for long time periods. More briefly, emitting variable bit rate caused FIB outperforms BEB at some FIB series ranges. This is because adopting BEB sounds to be unfair as nodes emitting 
light traffic load will need to wait unreasonable long time for those nodes which have high traffic load and already reached high $\mathrm{BE}$ values to finish transmitting their data. The problem becomes worse in frequent and traffic load networks. However, the incremental nature of FIB series up to a certain value after which it will reinitialized and start counting all over again gives a chance for a high fraction of nodes choose small and different waiting backoff periods and hence improving network performance.

As a future work, a dynamic FIB algorithm will be developed through which network changes its Fibonacci sequence maximum range dynamically according to its density and traffic load. For infrequent traffic load, it can work through high FIB range, whereas for active and frequent traffic, choosing small FIB range would be more sufficient. Another future work is to propose an adaptive BEB-FIB backoff algorithm through which nodes can dynamically change their backoff scheme according to the data traffic type and load. Nodes transmit data intensively can choose FIB and can convert to BEB as their load diminish, whereas those which emit lower constant traffic load can choose BEB backoff. Also, an enhancement on FIB algorithm will be achieved by proposing a hybrid BEB-FIB backoff algorithm which investigates the randomness property of BEB along with the FIB incremental feature which could eliminate the possibility of nodes choose or reach same backoff periods.

\section{References}

[1] Anastasi G., Conti M., and Di Francesco M., "A Comprehensive Analysis of the MAC Unreliability Problem in IEEE 802.15.4 Wireless Sensor Networks", IEEE Transactions on Industrial Informatics, Vol. 7, Issue 1. Pp. 52 - 65. February 2011. http://dx.doi.org/10.1109/TII.2010.2085440

[2] Buratti C., "Performance Analysis of IEEE 802.15.4 Beacon-Enabled Mode", IEEE Transactions on Vehicular Technology, Vol. 59, Issue 4. Pp. 2031 - 2045. May 2010. http://dx.doi.org/10.1109/TVT.2010.2040198

[3] Rao V., and Marandin D., "Adaptive Backoff Exponent Algorithm for Zigbee (IEEE 802.15.4)", Next Generation Teletraffic and Wired/Wirless Advanced Networking, Vol. 4003, Pp. 501 - 516. 2006. http://dx.doi.org/10.1007/11759355_46

[4] Manaseer S., Ould-Khaoua M. and Mackenzie L., "Fibonacci Backoff Algorithm for Mobile Ad Hoc Networks", DCS Technical Report Series Dept of Computing Science, 2006.

[5] Lee H., Lee K., and Ryu S., Lee S., Song K., Shin Y., “An efficient slotted CSMA/CA algorithm for the IEEE 802.15.4 LR-WPAN", International Conference on Information Networking (ICOIN 2011). Seoul, South Korea, January 26-18, 2011.

[6] Khan B., Ali F., and Stipidis E., "Improved Backoff Algorithm for IEEE 802.15.4 Wireless Sensor Networks”, Wireless Days (WD 2010), IFIP, Venis, Italy, October 20-22, 2010. http://dx.doi.org/10.1109/WD.2010.5657714

[7] Lee S., Shin Y., Ahn J. and Lee K., "Performance Analysis of a Non-Overlapping Binary Exponential Backoff Algorithm over IEEE 802.15.4”, Fourth International Conference on Ubiquitous Information Technologies and Applications (ICUT 2009). Fukuoka, December 20-22, 2009. http://dx.doi.org/10.1109/ICUT.2009.5405718

[8] Khanafer M., Guennoun M. and Mouftah H.,T., "Priority-Based CCA Periods for Efficient and Reliable Communications in Wireless Sensor Networks", Wireless Sensor 
$\begin{array}{lllllll}\text { Network, } & \text { Vol. 4, Issue 2. Pp 45-51. February } 2012 .\end{array}$ http://dx.doi.org/10.4236/wsn.2012.42007

[9] Khanafer, M., Guennoun M. and Mouftah, H.T.,"An Efficient Adaptive Backoff Algorithm for Wireless Sensor Networks", IEEE Global Telecommunication s Conference (GLOBECOM 2011), Houston, TX, USA, December 5-9, 2011. http://dx.doi.org/10.1109/GLOCOM.2011.6134430

[10]Lee B.-H. and Wu. H.-K., "Study on a delayed backoff algorithm for IEEE 802.15.4 low-rate wireless personal area networks", Communications IET, Vol. 3, Issue 7. Pp. 1089-1096. July 2009. http://dx.doi.org/10.1049/iet-com.2007.0517

[11]Wong C.-M. and Hsu W. -P "An Additional Clear Channel Assessment for IEEE 802.15.4 Slotted CSMA/CA Networks", IEEE International Conference on Communication Systems (ICCS 2010), Singapore, November 17-19, 2010. http://dx.doi.org/10.1109/ICCS.2010.5685903

[12] Woo S., Park W., Ahn S., An S. and Kim D., "Knowledge-Based Exponential Backoff Scheme in IEEE 802.15.4 MAC", in Information Networking. Towards Ubiquitous Networking and Services, Vol. 5200, Springer-Verlag Berlin, Heidelberg, Park, pp 435 444. http://dx.doi.org/0.1007/978-3-540-89524-4_44

[13] Ko J.-G., Cho Y.-H., Kim H., "Performance Evaluation of IEEE 802.15.4 MAC with Different Backoff Ranges in Wireless Sensor Networks", $10^{\text {th }}$ IEEE Singapore International Conference on Communication Systems (ICCS 2006), Singapore, October, 2006. http://dx.doi.org/10.1109/ICCS.2006.301525

[14]Z. J. Haas, and Deng J., "On Optimizing the Backoff Interval for Random Access Schemes”, IEEE Communications, Vol. 51, Issue 12. Pp 2081 - 2090. December 2003. http://dx.doi.org/10.1109/TCOMM.2003.820754

[15]Ha J. Kim T.H., Park H., Choi S., Kwon W., "An Enhanced CSMA-CA Algorithm for IEEE 802.15.4 LR-WPANs", IEEE Communications Letter, Vol. 11, Issue 5. Pp 461 463. May 2007.

[16] Yanliang J., Huijun M., Haitao L. Yong X., “An Improved Backoff Algorithm for Wireless Sensor Network Based on Game Theor", International Communication Conference on Wireless Mobile and Computing (CCWMC 2009) IET, Shanghai, China, December 7-9, 2009.

[17] Song J., Shin k.-Y., Yu M., Kim J., Mah P., "NACA: A New Adaptive CSMA/CA Algorithm of IEEE 802.15.4 in Beacon-enabled Networks", $9^{\text {th }}$ International Conference on Advanced Communication Technology (ICACT 2007), Gangwon-Do, Febuary 12-14, http://dx.doi.org/10.1109/ICACT.2007.358352

[18] Yassein M., Manaseer S., and Al-Turani A., "A Performance Comparison of Different Backoff Algorithms under Different Rebroadcast Probabilities for MANET's”, 25th UK Performance Engineering Workshop, Leeds, UK, July 6-7, 2009.

[19] Manaseer S., Bani Yassein M. and Momani A., "A History-Based Backoff Algorithm for Mobile Ad Hoc Networks", The $2^{\text {nd }}$ International Conference on Information and Communication Systems (ICICS 2011), Irbid, Jordan, May 22-24, 2011.

\section{Copyright Disclaimer}

Copyright reserved by the author(s).

This article is an open-access article distributed under the terms and conditions of the Creative Commons Attribution license (http://creativecommons.org/licenses/by/3.0/). 